\title{
Machine learning in medicine
}

\author{
Chloe Gui, Victoria Chan \\ Faculty Reviewer: Daniel J Lizotte, MSc, PhD (Department of Computer Science)
}

\section{ABSTRACT}

Machine learning (ML) is a powerful and flexible tool that can be used to analyze and predict outcomes from biological and clinical data. ML models have the potential to improve healthcare efficiency in a number of ways. Algorithms that predict prognosis empower healthcare officials to allocate resources optimally and physicians to select better treatment options for patients. Diagnostic models can be used in screening, in risk stratification, and to recommend appropriate testing and treatment. This would decrease the burden on physicians, increase and expedite patient access to care, save resources, and reduce costs. However, despite the research advances of ML in medicine, its role in the clinic is currently limited. Model building and validation may require large amounts of high-quality data that can be difficult and expensive to obtain, and diagnostic models must be individually built for each disease, a lengthy process. The psychological aspect of trusting black box algorithms may also be challenging to accept. Continued ML research, however, may enable the use of smaller datasets and the development of more transparent models. Careful trials in the clinic will need to be conducted before the more impactful uses of ML, such as diagnosis, can be implemented.

\section{INTRODUCTION}

Machine learning (ML) is a branch of artificial intelligence (AI) that has become ubiquitous in many fields. Essentially, from an initial training dataset of features and outcomes, an algorithm learns how the features relate to and are predictive of the outcomes. On subsequent data, the ML model can predict outcomes when presented only with the features..$^{1-4}$ Models are currently used by banks to detect fraudulent transactions, ${ }^{1}$ by email servers to filter spam, ${ }^{2}$ and by astronomers to analyze galaxy images. ${ }^{3} \mathrm{ML}$ especially excels at identifying patterns in large and noisy datasets, making it useful for analyzing complex biological data. As a simple example, an algorithm may be trained to predict outcomes such as whether a tumour is benign or malignant from features such as tumour size. The model could then make predictions on new tumours whose outcomes are unknown. Data on which ML can be trained range from numerical data of blood biomarkers to medical images. Thus, ML is a versatile and powerful tool that potentiates personalized medicine, providing a more precise understanding of individual patients and their needs. Here, we examine how ML can improve the efficiency of our healthcare system in areas of prognostics, diagnostics, and increasing access to medical care.

\section{PREDICTING PROGNOSIS}

Prognosis prediction includes approximating outcomes such as a patient's disease susceptibility, disease recurrence likelihood, life expectancy, and response to treatment. The factors involved are complex and multifactorial, and thus, it is difficult to provide a definitive prognosis for many conditions., ${ }^{5,6}$ Accurate prognosis prediction is valuable as it helps healthcare providers make informed decisions about resource allocation and best treatment practices.? A tool to predict response to treatment, for instance, would allow physicians to tailor treatments to individual patients. Such a tool would enable physicians to identify which patients would benefit from adjuvant or alternative therapies and which patients would not, sparing the latter from treatment risks and side effects.

The field of oncology in particular has been extensively studying the use of ML methods in predicting prognosis. One review reported that ML improves cancer prognostic predictions by $15-25 \%{ }^{8}$ The rise of high-throughput sequencing has pushed the consideration of genetic markers in treatment decisions, and ML is useful for modeling complex genetic data. For example, a classification ML algorithm trained on microarray data of breast tumour tissues found different genetic signatures associated with metastatic phenotypes and with certain treatment prognoses. ${ }^{9}$ These patterns could serve as clinical indications for different treatments, leading to better patient outcomes. A study on hepatitis B virus-positive metastatic hepatocellular carcinoma also used ML to identify signatures from 153 genes to predict metastasis and survival. ${ }^{10}$

\section{SCREENING, DIAGNOSIS, AND ACCESS TO CARE}

$\mathrm{AI}$ has the potential to streamline healthcare diagnostics by reducing cost and time to diagnosis. ML has been demonstrated to be capable of screening patients, stratifying patients by risk, and assisting physicians in decision making. Screening models have been built to detect diseases such as congenital cataracts, ${ }^{11}$ skin cancer ${ }^{12}$ heart disease, ${ }^{4}$ hepatitis disease,${ }^{13}$ and autism. ${ }^{14}$ Given the high stakes of medical decisions, a model built with particularly high sensitivity could be an inexpensive tool to rule out diagnoses, leaving potentially positive cases for physicians to investigate. These models could also be distributed to patients for preliminary evaluation and to prioritize patients at risk. For example, Esteva et al trained algorithms to classify skin lesion images as cancerous or benign at an accuracy that matched dermatologists. ${ }^{12}$ Mobile-based evaluation of other diseases could also be feasible. For instance, Wall et al developed a highly accurate model to assess and diagnose autism. ${ }^{14}$ Given that the incidence of autism is increasing and evaluation involves analysis of candidates' home videos, mobile tools could be valuable in assessing a wider population. ${ }^{14}$ Indeed, models developed for many diseases could potentially be deployed on mo- 
bile devices for greater access to care.

Screening could be particularly advantageous for rare diseases. Rare diseases are typically difficult to identify, and this may lead to delayed or incorrect treatment, possibly with harmful consequences to the patient. Because specialists are usually concentrated at larger health institutes, patients with rare diseases may have difficulty accessing expertise for diagnosis and care. ${ }^{11}$ Thus, Long et al examined whether AI could provide a unique management system for rare diseases. They built ML models to screen patients for congenital cataracts, perform risk stratification, and suggest treatment. In fact, the models performed to the same level as ophthalmologists. ${ }^{11} \mathrm{~A}$ widely accessible system of such models could increase access to the expertise required to identify and care for patients with rare diseases.

\section{CHANGING THE ROLE OF THE PHYSICIAN}

As ML algorithms improve and their applicability to healthcare broadens, ML will be gradually introduced to the healthcare system. In fact, ML already forms the basis of radiology tools such as image segmentation to isolate areas of interest. ${ }^{15}$ Increased capabilities of ML, however, may displace physicians from some of their roles. For instance, image-reading specialties such as radiology involve identifying patterns from medical images. Such tasks are suitable for ML as the data are consistent in format, and diagnoses often can be made from the images alone. Given equal accuracy to human assessment, algorithms would be advantageous in providing immediate results while reducing cost. One study, for instance, found that computer-aided detection could replace the traditional second reader in detecting small breast cancers. ${ }^{16}$ The increasing use of imaging due to technological advances has also escalated the workload for radiologists. ${ }^{17} \mathrm{ML}$ would reduce the burden and provide consistent 24-hour service, whereas human radiologists may make errors in circumstances such as overnight shifts. Models that have been built for computer-aided diagnosis include those that detect pulmonary embolisms, ${ }^{14}$ polyps in CT colonography, ${ }^{15}$ and patterns of mild cognitive impairment in brain scans that precede $\mathrm{Alz}$ heimer's disease..$^{15}$ By extension, physicians of other image-based specialties, such as pathology, may also perform fewer image analysis tasks as ML algorithms improve.

\section{CHALLENGES TO TRANSLATION}

Despite its widespread use in other fields, ML has failed to make an equivalent impact in the healthcare system. One of the biggest challenges is the enormous amount of high-quality data often required to build and validate ML models. Though patient data is increasing in abundance, they may be incomplete for ML purposes. One model that aimed to predict the survival of postmenopausal women using electrocardiogram biomarkers, clinical factors, and demographic variables performed worse than the current standard, the Framingham Risk Score. ${ }^{18}$ This was at least partly because the data lacked information known to be important to the outcome, such as blood biomarkers. Another possible problem in applying ML to healthcare data is that predictive variables may be infre- quently tested in clinic or only tested in limited patient subgroups and thus are generally unavailable. Therefore, there may exist important predictive features that ML would fail to recognize. Furthermore, some lesions, such as in cardiac and vascular tissues, are generally not biopsied, which limits the availability of molecular and histological information. ${ }^{19}$ Aside from the data needed to build the model, data must also be acquired from multiple independent populations to validate the model. As biological data is expensive to acquire and not typically shared among researchers, quality and quantity of information is a major inhibitor of ML progress in medicine. The study that used electrocardiogram markers to predict mortality in postmenopausal women, for instance, failed to find an appropriate external dataset on which to validate their model. ${ }^{18}$ These issues are currently being addressed by research that aims to build models from smaller datasets. ${ }^{20}$

Another challenge to translation is the psychology involved in using machines to manage patients. A human error rate is generally accepted in healthcare, but machine error that carries health consequences may be difficult to accept. Some powerful ML methods are also black box models where the algorithmic mechanism is unknown, and the medical community may feel uncomfortable with this lack of transparency. However, ongoing research efforts to improve model transparency are promising, ${ }^{21}$ and if $\mathrm{ML}$ performance eventually exceeds that of a physician for a task, it may be considered inefficient and unethical not to defer to ML. Additional clinical trials will be required to determine the appropriate and responsible use of $\mathrm{AI}$ in healthcare.

\section{CONCLUSION}

ML shows tremendous promise in increasing the efficiency of our healthcare system. It has demonstrated the ability to predict prognoses, provide diagnoses, and increase the reach of medical care. As novel predictors of health-related outcomes are discovered, ML findings will also contribute to research developments and our understanding of disease. However, because ML generally requires large quantities of high-quality data, progress is costly and time-consuming. Nonetheless, advances suggest that this technology may grow to be a fundamental part of our healthcare system, decreasing the burden on physicians and improving the speed and depth to which patients can be attended. As domain knowledge is necessary to interpret ML findings, physicians will continue to be key in providing empathetic care.

\section{REFERENCES}

1. Perols J. Financial statement fraud detection: An analysis of statistical and machine learning algorithms. Auditing-J Pract Th. 2011 May;30(2):19-50.

2. Guzella TS, Caminhas WM. A review of machine learning approaches to spam filtering. Expert Syst Appl. 2009 Sep;36(7):10206-222.

3. Kuminski E, George J, Wallin J, et al. Combining human and machine learning for morphological analysis of galaxy images. Publ Astron Soc Pac. 2014 Oct;126(944):959-67.

4. Magoulas GD, Prentza A. Machine Learning and Its Applications. Berlin Heidelberg: Springer; 2001 Sep. 300-7 p. 
5. Christakis NA, Lamont EB. Extent and determinants of error in physicians' prognoses in terminally ill patients. BMJ. 2000 Feb;320(7233):469-72.

6. Clément-Duchêne C, Carnin C, Guillemin F, et al. How Accurate Are Physicians in the Prediction of Patient Survival in Advanced Lung Cancer? Oncologist. 2010 Jun;15(7):782-9.

7. Ohno-Machado L. Modeling Medical Prognosis: Survival Analysis Techniques. J Biomed Inform. 2001 Dec;34(6):428-39.

8. Kourou K, Exarchos TP, Exarchos KP, et al. Machine learning applications in cancer prognosis and prediction. Comput Struct Biotechnol J. 2014 Nov;15(13):8-17.

9. Van ‘t Veer LJ, Dai H, Van de Vijver MJ, et al. Gene expression profiling predicts clinical outcome of breast cancer. Nature. 2002 Jan;415(6871):530-536.

10. Ye QH, Qin LX, Forgues M, et al. Predicting hepatitis B virus-positive metastatic hepatocellular carcinomas using gene expression profiling and supervised machine learning. Nat Med. 2003 Apr;9(4):416-23.

11. Long E, Lin H, Liu Z, et al. An artificial intelligence platform for the multihospital collaborative management of congenital cataracts. Nat Biomed Eng. 2017 Jan;1(1):0024.

12. Esteva A, Kuprel B, Novoa, RA. Dermatologist-Level Classification of Skin Cancer With Deep Neural Networks. Nature. 2017 Jan;542(7639):115-8.

13. IEEE Neural Networks Society. Proceedings of the International Joint Conference on Neural Networks, 2003. 2003 Jul 20-24; Portland, OR. New York City: IEEE; Aug 2003.

14. Wall DP, Kosmicki J, DeLuca TF, et al. Use of machine learning to shorten observation-based screening and diagnosis of autism. Transl Psychiatry. 2012 Apr;2:e100.

15. Wang S, Summeres RM. Machine learning and radiology. Med Image Anal. 2012 Jul;16(5):933-51.

16. Gilbert FJ, Astley SM, Gillian MGC, et al. Single Reading with Computer-Aided Detection for Screening Mammography. N Engl J Med. 2008 Oct;356:1675-84.

17. Smith-Bindman R, Miglioretti DL, Johnson E, et al. Use of diagnostic imaging studies and associated radiation exposure for patients enrolled in large integrated health care systems, 1996-2010. JAMA. 2012 Jun;307(22):2400-9.

18. Gorodeski EZ, Ishwaran H, Kogalur UB, et al. Use of hundreds of electrocardiographic biomarkers for prediction of mortality in postmenopausal women: the Women's Health Initiative. Circ Cardiovasc Qual Outcomes. 2011 Sep;4(5):521-32.

19. Deo RC. Machine Learning in Medicine. Circulation. 2015 Nov;132(20):1920-30.

20. Boulicaut JF, Esposito F, Giannotti F, et al. Knowledge Discovery in Databases: PKDD 2004. 8th European Conference on Principles and Practice of Knowledge Discovery in Databases; 2004 Sep 20-24; Pisa, Italy. Berlin Heidelberg: Springer; 2004 Sep. 161-172 p.

21. Henao R, Lu JT, Lucas JE, et al. Electronic Health Record Analysis via Deep Poisson Factor Models. 2016 Apr;17(186):1-32. 